\title{
Anomalous Self- and World Experiences in Depersonalization Traits: a Qualitative Study
}

\author{
Anna Ciaunica ${ }^{1,2^{*}}$, Elizabeth Pienkos ${ }^{3^{*}}$, Estelle Nakul ${ }^{4}$, Luis Madeira ${ }^{5}$, Harry Farmer
}

\section{Corresponding Authors:}

\author{
Dr. Anna Ciaunica* - a.ciaunica@ucl.ac.uk | \\ Dr. Elizabeth Pienkos* - epienkos@clarkson.edu
}

\begin{abstract}
Affiliations
1 Institute of Philosophy, University of Porto, via Panoramica s/n 4150-564, Porto, Portugal |

2 Institute of Cognitive Neuroscience, University College London, WC1N 3AR, London, UK

3 Department of Psychology, Clarkson University, Potsdam, NY, USA

4 Aix Marseille Univ, CNRS, LNC, FR3C, Marseille, France

5. Faculdade de Medicina, Universidade de Lisboa, Lisboa, Portugal

6. School of Human Sciences, University of Greenwich, UK
\end{abstract}

\section{Abstract}

This paper proposes a qualitative study exploring anomalous self and world-experiences in individuals with high levels of depersonalization traits. Depersonalization (DP) is a condition characterized by distressing feelings of being a detached, neutral and disembodied onlooker of one's mental and bodily processes or even of reality itself ('derealization'). Feelings of depersonalization are extremely common in the general population, yet under-acknowledged and under-examined.

Our findings indicate the presence of a wide range of anomalous experiences traditionally understood to be core features of depersonalization, such as disembodiment and disrupted self-awareness. However, our results also indicate experiential features that are less highlighted in previous work, such as faster time perception and blurriness of the self/other boundaries which may play a key role in altering one's sense of self and sense of presence in the world.

Our qualitative study provides an in-depth examination of self-reported disturbances of one's relatedness to one's self and the world, thereby shedding further light on the nature of altered subjective experiences in DP. In doing so, this paper draws attention to key aspects yet overlooked that may prove valuable for potential diagnosis and therapy. We conclude by highlighting limitations of this study and a number of open questions that further work needs to address in the future, in order to better understand this condition and to improve the quality of life of those experiencing depersonalization.

Funding info - This work was supported by a Bial Foundation grant $n^{\circ} 157 / 16$ to AC \& HF 


\section{$\S 1$ Introduction}

In daily life, our experiences seem to be tacitly infused by a sense of self - the feeling that there is a "real me" or "self" intimately linked to my body as the subject of sensations, experiences and thoughts, and which is distinct from the rest of the world and others. In addition, we also seem to experience a sense of presence or "realness"- the feeling that there is a "real world" out there, with which I can fully engage, exchange and interact.

A significant body of work across disciplines and theoretical frameworks outlined that our sense of self is a fundamental aspect of our conscious experiences (Merleau-Ponty 1962; Gallagher 2000; Damasio 2000; Sass and Parnas 2003; Zahavi 2005; Blanke and Metzinger 2009; Hohwy 2007; Ciaunica and Fotopoulou 2017; Friston 2017) (see also Quin et al. 2020 for a recent review). But what if instead of being the first-person subject of my perceptions, emotions and actions, I constantly endorse the third-person perspective of an 'external' observer on my own experiences? What if I feel unreal, non-present, walking through daily life like an automaton, or like in a dream?

Depersonalization (DP henceforth) is a condition characterized by distressing feelings of being a detached, neutral and disembodied onlooker of one's mental and bodily processes or even of reality itself ('derealization') (Sierra \& Berrios 1998). This profound disruption ${ }^{1}$ in the sense of self affects equally (a) the sensory and bodily aspects of the self (detachment from one's body or body parts); (b) the experiential aspects (detachment from one's subjective feelings and emotions); and (c) the cognitive and narrative aspects (disconnection from one's personal stories, memories, thoughts and future plans), often described by DP sufferers as a lack of a narrative flow or a 'plot' in one's life. (Ciaunica et al. 2020).

DP has a prevalence of around 1-2\% of the population (Hunter et al. 2004; Michal et al. 2007; Lee et al. 2012), which is comparable to that of schizophrenia for example. Also, the prevalence of transient episodes of depersonalization is between 34 and $70 \%$ in the general population (Aderibigbe et al. 2001; Hunter et al. 2017). Transient occurrences of depersonalization episodes have been reported by almost $50 \%$ of college students (Dixon, 1963). Everyday phenomena such as fatigue (Tibubos et al., 2018), sleep deprivation (van Heugten-van der Kloet et al., 2015), or travelling to new places may also trigger transient depersonalization feelings (Kaplan et al., 1998) (see Salami et al. 2020 for a recent discussion). However, it currently takes an average 7 to 12 years to correctly identify and diagnose DP, which makes it one of the most prevalent yet under-diagnosed conditions (Hunter et al. 2017; Michal et al. 2010).

There are at least three main reasons for this significant delay and misperception in diagnosing DP. First, from a practical point of view, there is a lack of awareness regarding DP among

\footnotetext{
1 When these phenomena become debilitating and chronic as dominant symptoms, they are diagnosed as depersonalization-derealization disorder (DPD) in DSM5, grouped among the dissociative disorders (APA, 2013). DPD has been characterized as involving four main experiential components: (1) feelings of disembodiment (lack of body ownership and/or agency feelings), (2) emotional numbing (altered ability to attribute to perceived objects or concrete situations with emotional feeling), (3) anomalous subjective recall ([a] the "factual" aspect of memory is preserved but without the feeling the event really happened and $[b]$ disturbances in the capacity for imagery), and (4) derealization (detachment, feeling cut-off from the world, things seem unreal) (Sierra \& David 2011).
} 
medical practitioners (Medford et al. 2005). Second, there is important overlap between the phenomenology of DP symptoms and other conditions such as depression, post-traumatic stress disorder, anxiety disorder and migraine (Devinski et al 1989; Lambert et al. 2002; Armour et al. 2014). For example, transient DP experiences are reported in several major conditions such as (i) anxiety (Baker et al. 2003), (ii) panic attacks (Harper and Roth 1962), (iii) post-traumatic stress disorder (Serra et al. 2007; Lanius et al. 2012), (iv) burnout syndrome (Mayer-Gross 1935; Masiach and Jackson 1981), (v) after prolonged sleep (Bliss 1959), (vi) hallucinogenic toxicosis (Fleming 1936), (vii) severe melancholic episodes (Kraus 2002; Fuchs 2005), and (viii) schizophrenic passivity experiences (Langfeldt 1960). Finally, DP experiences and symptoms overlap with other well-known dissociative conditions such as schizophrenia and psychosis.

In the light of this significant prevalence in the general population, combined with the lack of appropriate and rapid diagnosis tools, it is important to delineate the phenomenological markers that underlie DP experiences. This is because when people start to experience these phenomena, they typically report them to close and trusted people, as well as to general practitioners. In other words, they use self-reports. There is thus an urgent need to better examine and understand DP experiences as reported from a first-person perspective. Qualitative studies have the potential to provide precious insights into the key aspects of phenomena as subjectively lived by the individual.

The current paper proposes a qualitative study exploring anomalous self and world-experiences that occur in individuals who experience high levels of DP traits. It aims at providing an in-depth examination of self-reported disturbances of one's relatedness to one's self and the world, thereby shedding further light on the nature of altered subjective experiences in DP.

In doing so, we build upon two influential qualitative research tools, namely (1) the Examination of Anomalous Self-Experience (EASE) (Parnas et al., 2005) and (2) Examination of Anomalous Self-Experience (EAWE) (Sass et al., 2017). The EASE and EAWE are two semi-structured phenomenological interviews derived from self-reports, clinical descriptions and interviews as well as empirical and theoretical studies designed to tackle disturbances in the lived self and world (though not necessarily exclusive to) schizophrenia spectrum conditions ${ }^{2}$. The EASE emphasizes disturbances in self-experience, whilst the EAWE is intended to investigate disturbances of the world-experiences. While the EASE/EAWE have the advantage of providing a comprehensive assessment of minimal self-disturbances, they may be also resource-intensive instruments, requiring significant training and potentially lengthy interview times. Hence, in some research situations, self-reports in shorter versions might be considered as an alternative option.

For example, the Inventory of Psychotic-Like Anomalous Self- Experiences (IPASE) (Cicero, et al., 2017) was developed within the tradition of objective scale development (Clark \& Watson, 1995) in which an overinclusive item-pool was established and items were retained or excluded based on their performance on exploratory and confirmatory factor analyses. Preliminary data

\footnotetext{
2 The interviews have been refined through clinical trials and with input from "experts by experience" (persons with schizophrenia and other psychoses) and clinicians familiar with phenomenological approaches to schizophrenia.
} 
indicates that the IPASE demonstrates strong convergent and discriminant validity, and that it may be used as an effective screener for disturbances of minimal selfhood (Nelson et al., 2018). Recently, a new tool has been proposed, namely the Examination of Anomalous World Experience-Self Report (EAWE-SR). The EAWE-SR is a qualitative self-report tool developed to specifically reflect the content of the EAWE, with minor wording and organization changes to improve clarity and comprehensibility by participants without a background knowledge of phenomenology. Its psychometric properties have not yet been established at the time of the writing of this paper.

Taken together, these validated and innovative self-report tools offer an extremely comprehensive assessment of qualitative alterations in the experience of self and world. For this reason, they were adopted for this study as a means of eliciting descriptions of anomalous selfand world experiences among persons with high DP traits in a self-report format. Our study offers precious new insights into what it is like to live with depersonalization from a first-person perspective. The self-reports that we have collected and analyzed here unveil core aspects of the phenomenological markers underlying anomalous self- and world-experiences in depersonalization. These indicators may thus significantly advance our understanding of the altered sense of self and sense of realness in DP. They can also be used in designing potential diagnosis tools for describing depersonalization, a widely spread yet under-diagnosed condition.

Our paper proceeds as follows. In Sections 2 and 3 we describe in detail the Methods that we used in our study, the qualitative themes, as well as our Results. Section 4 provides a general discussion of our findings. Finally, in Section 5 we briefly present the limitations, conclusions and future directions for further research in this important and neglected area.

\section{§ 2 Methods}

\subsection{Sample}

A total number of 24 participants (18 females and 6 males) participated in this study. The average age of the participants was 23.3 years ( $(\mathrm{sd}=4.23$ years). Participants were recruited from previous studies conducted by members of this research group. Participants were invited to participate if they had high trait DP as determined by scores of higher than 50 on the Cambridge Depersonalization Scale (CDS-29; Sierra \& Berrios, 2000) at the time of previous testing, and if they agreed to be contacted for additional studies.

Participants were contacted using the research institute database, university subject pool, and university participant mailing channels. Initial screening for DP was completed using an online version of the 2-item form of the Cambridge Depersonalisation Scale (CDS-2), comprising the statements 'My surroundings feel detached or unreal, as if there was a veil between me and the outside world' and 'Out of the blue, I feel strange, as if I were not real or as if I were cut off from the world', scored on a 4-point Likert scale from 0 ('not at all') to 3 ('nearly every day'), relevant to the past two weeks. There were four additional screening questions ('Do you have nightmares more than 4 times/week?', 'Are you taking antidepressants or antipsychotics?', 'Do you see colours when you listen to music?', and 'Are you suffering from seizure disorder?'). 
Respondents from the survey were invited to take part if they scored 3 or higher on the CDS-2, and 0 on the distractor questions (high-depersonalisation group). All invited respondents then completed the full CDS (29 items) ${ }^{9}$ to measure frequency and duration of depersonalisation symptoms within the last 6 months. The CDS contains statements describing abnormal sensory experiences and somatosensory distortions, inability to experience some emotions, heightened self-observation, autoscopy and out-of- body experiences, abnormal body ownership and lack of agency (Sierra et al., 2005). The frequency of these experiences has been measured on a 5-point Likert scale (never 0, rarely 1, often 2, very often 3, all the time 4), and their duration on a 6point scale (few seconds 1 , few minutes 2 , few hours 3 , about a day 4 , more than a day 5 , more than a week 6). All scores were added to compute each participant's global depersonalization score (0-290). Participants with a score higher than 50 were assigned to the High DP group, and were invited to take part in this study. Additional eligibility criteria included: (a) fluent in English; (b) between the ages of 18 and 40; (c) no history of neurological illness. The CDS also provides the following subscores: Anomalous Body Experience (ABE), Anomalous Subjective Recall (ASR), Emotional Numbing (EN) and Alienation from Surroundings (AfS). These were used to assess the relation between CDS scores and IPASE scores.

All participants were given a unique code and provided written informed consent prior to any data collection. Participants then completed all study measures online (using their own devices to complete the measures). Participants were notified before completing the measures that they could stop at any time, that they could take breaks as needed and that they could withdraw from the study at any point. Participants were also given the information to contact a crisis centre or hotline prior to and after completing study measures as well as a link to information on depersonalization.

All information was pseudo-anonymized by study personnel to protect the identity of participants. Study oversight was provided by the overseeing institution's ethics board.

\subsection{Study measures}

Study measures included the Inventory of Psychotic-like Anomalous Self-Experiences (IPASE) and the Examination of Anomalous World Experience-Self Report version (EAWE-SR). The IPASE (Cicero et al., 2017) is a 57-item self-report scale that was designed to quickly assess a subject's level of anomalous self-experience. It was developed by generating an overinclusive list of questions thought to capture disturbances of selfhood, which was then refined based on responses from large subject pools. Exploratory factor analysis has revealed five unique factors reflecting disturbances in Cognition, Consciousness, Self-Awareness and Presence, Somatization, and Transitivism/Demarcation. Each item includes five possible responses, which are scored from 1-5: Strongly disagree (1 point), Disagree (2 points), Neither agree nor disagree (3 points), Agree (4 points), and Strongly agree (5 points). Total scores on the IPASE may range from 57 to 285 . Past studies have demonstrated strong psychometric properties, including correlation with high levels of positive schizotypy and schizophrenia, groups that are thought to also have high levels of self-disturbance (Cicero et al., 2017). For the purposes of retrieving qualitative information, an additional open-ended response was added to each item: What is the 
experience like for you? Please provide any details you think are relevant to understanding this experience.

The EAWE-SR (unpublished) is a 210-item self-report instrument that was developed on the basis of the EAWE interview (Sass et al., 2017). It assesses six domains of anomalous world experience: Space and objects, Time and events, Other persons, Language, Atmosphere and Existential orientation. Each item includes four possible responses, which are scored from 1 to 4 : I have never experienced this (1 point), I have only experienced this occasionally ( 2 points), I have experienced this frequently (3 points), and I experience this every day or constantly (4 points). Scores may range from 210 to 840 . In this study, all items also contained the following open-ended response option: What is the experience like for you? Please provide any details you think are relevant to understanding this experience. As this instrument is still in development, no psychometric data is available; it was used solely for the purpose of eliciting qualitative data about a large range of potential changes in experiences of the external world.

\subsection{Data Analysis}

Basic descriptive statistics (mean, standard deviation, range) for both the IPASE and the EAWESR were calculated. As the psychometric properties for the EAWE-SR have not yet been established, descriptive data are presented for informational purposes only.

Qualitative data were analyzed using inductive thematic analysis (Braun \& Clark, 2006). This approach to data analysis is used to organize similar pieces of data around different themes that emerge from the data itself. In this study, it involved several steps: familiarizing oneself with the data, generating initial codes, searching for themes, reviewing themes, defining and naming themes, and producing the report. In this study, two co-authors ( $\mathrm{XX}$ and $\mathrm{XX}$ ) reviewed portions of the data and provided reflections on data meaning. Following this, one co-author (EP) developed codes based on the data and reflections and used these to generate themes. Responses were not coded when they were clearly describing experiences that only occurred in the context of substance use, a medical condition, sleeping/waking up, as the result of imagination or playful entertainment of ideas or if they reflected very typical experiences according to at least two authors who reviewed the data. All themes were then reviewed by the first author, with changes made as necessary to ensure coherent and cohesive themes. Themes were then defined, and themes and coding scheme were sent to co-authors (AC, EN, and LM) to review and provide feedback on appropriateness of code and theme identification, and suggest changes as necessary. These suggestions were reviewed and any discrepancies or disagreements were discussed by coders to arrive at consensus.

\section{§ 3 Results}

\subsection{Descriptive Statistics}

The means of total scores on study measures are reported in Table 1. Some CDS scores were lower than those reported during the initial screen for the study - and indeed, some scores fell 
below the initial cutoff of 50 points. However, other scores were higher, suggesting that any changes in scores represented natural fluctuations of DP experiences over time. All data was kept for this study, as the primary goal was to include participants who had experienced high levels of DP at some point in their lives (though not necessarily at the time of data collection).

\begin{tabular}{|l|l|l|}
\hline Measure & Total score (sd) & Range \\
\hline CDS (start of study) & $63.75(25.84)$ & $17-115$ \\
CDS (pre-study screen) & $73(25.01)$ & $51-151$ \\
\hline IPASE (total score) & $123.33(31.79)$ & $71-180$ \\
IPASE (item score) & $2.16(0.56)$ & \\
\hline EAWE-SR (total score) & $291.79(52.56)$ & $215-410$ \\
EAWE-SR (item score) & $1.39(0.25)$ & \\
\hline
\end{tabular}

Table 1. Means and range of study measures

\subsection{Qualitative Themes}

The following themes reflect a diverse range of experiences reported by the participants in this study in response to the open-ended questions added to the IPASE and the EAWE-SR. Each theme comprises a number of related codes, which are also listed. Detailed descriptions of the codes and illustrative examples are provided in the supplementary material. The number of participants who describe such changes are listed in parentheses.

\subsubsection{Detachment (22 participants)}

Many participants described feelings of being detached from the world and from oneself, involving a sense of not being fully engaged with or thrown into the world and one's own experience. The clearest examples of detachment involved a general sense of not being fully present to oneself, of feeling like an observer of oneself or the world, of not feeling a sense of ownership over one's own thoughts and experiences, or of being detached from or not a part of the world. Other experiences included in this theme were feelings of objects or places being unfamiliar, or simply marveling at their existence, and of the world feeling unreal or lacking in vibrancy or depth. It also includes feelings of déjà $v u$, as though reliving a moment rather than living fully in the moment. (It is worth noting that some participants reporting déjà vu appeared to have experiences similar to the general population; though for some, these feelings could be quite strong and confusing.) Some participants noted that these feelings appeared to occur primarily in situations in which they were feeling depressed or anxious, though others did not attribute their experiences to particular psychological states.

\subsubsection{Embodiment (14 participants)}

There were many descriptions of unusual or changing experiences of the body. These could involve a fairly profound feeling that the body is disconnected from or not belonging to one's self, feeling as though one's expression or the body is out of one's control (e.g. feeling unable to 
move the body intentionally, or the body moving without apparent intention to do so), that it has changed or is in the process of changing inexplicably, that the participant is experiencing physical sensations that do not seem to have a clear physical cause, or sudden and inexplicable feelings of bodily weakness.

\subsubsection{Identity changes (19 participants)}

Participants described a lack of congruency or transparency related to one's sense of self. This could include a feeling of distance from oneself, an uncertainty about who one really is, a lack of congruency between one's felt self and one's thoughts or words or actions, or a feeling of disconnection or lack of continuity with one's past self. It also included feeling like one did not identify with one's age, feeling either older or younger than one actually is.

\subsubsection{Idiosyncratic beliefs (20 participants)}

Participants described holding several unique preferences, attitudes, beliefs, and values regarding their relationships, society, humankind and, most broadly, existence and reality itself. They described feelings of indifference toward others or humankind or the world as a whole (though note that some described a feeling of greater concern or responsibility for others, described below), a tendency to be skeptical of accepted aspects of reality, and a rejection of conventional attitudes or behaviors (either due to a preference for one's own values or dislike of conventional attitudes and behaviors), while others noted a preference for things and events to be orderly and regular. Some described a sense of having greater insight or awareness than most, a feeling of being guided or predestined in life, and a sense of encountering events that conveyed a special meaning to them. Several participants described anomalous ways of developing beliefs and ideas, including having greater openness to unusual ways of thinking about things or to seeing similarities or connections that others might not recognize, and a tendency to form beliefs without apparently sufficient evidence to support them. A number of participants also described beliefs about the end of the world being imminent, while some discussed a general awareness of a larger perspective than just their immediate view or that of the human race.

\subsubsection{Affective changes (21 participants)}

Participants described various forms of emotion and mood that were different from what the situation would typically elicit, including anxiety (which many described as occurring for no apparent reason), restlessness, and rapid shifts in emotions or emotions that are felt to be "inappropriate" to the situation. Related to this were specific experiences characterized by a sense of demoralization, including hopelessness, feelings of being unable to achieve one's goals or direct one's life, feeling like life is meaningless, overbearing feelings of fatigue, and not feeling strong emotional reactions or excitement about anything. One participant also described concerns about losing their sanity.

\subsubsection{Changes in communication and approach to language (18 participants)}

Participants noted numerous difficulties in expressing oneself clearly or fully to other people, or else the use of certain strategies to enhance clarity. This includes problems with finding the right 
word or clearly articulating oneself, lacking emotional intonation in speech, feeling unable to organize one's thoughts effectively in speech, or no longer wishing to speak. Some participants also described shifts in the way they think about, understand, or approach language itself. This includes a feeling of words being unable to convey experiences, various difficulties understanding speech or written language, feeling distracted by certain words, feeling like language and words are meaningless or absurd, or feeling like language has a certain degree of power or autonomy.

\subsubsection{Anomalous reactions to others (21 participants)}

Participants described several habitual and automatic reactions to other people. These might occur as a stylistic preference, a personality trait, or in response to reactions that others elicit. These experiences include rejecting some social norms (or preferring one's own norms), acting clownishly, acting in oppositional ways, and habitually avoiding the company of others (often due to feeling overwhelmed or exhausted). Others described feeling strongly influenced by others, at times intentionally imitating others' behaviors and mannerisms, or else feeling like their entire identity was shaped by other people. Some described acting in ways that were counter to their own preferences in order to be liked and to fit in or else in order to avoid conflict.

\subsubsection{Difficulty relating to others (21 participants)}

Participants described feelings of being disconnected or distant from other people, including feeling profoundly alone or isolated, either due to general feelings of distance and detachment, not understanding others, feeling the need to scrutinize and observe others' behavior to understand them, having to apply intellectualized strategies to understand and interact with others, not feeling truly known by others, feeling that others put on a mask or facade, or generally disliking other people. Some described feeling like people who were known to them were suddenly unfamiliar (to the extent, for one person, of doubting their past experiences with that person), feeling like others are lifeless or unreal, or feeling like some people are especially and unusually lively.

\subsubsection{Social anxiety and self-scrutiny (21 participants)}

Participants described numerous experiences of anxiety, fear, self-doubt, and self-criticism in social situations. These could occur during specific interactions with individuals, but they could also characterize an approach to other people as a whole. These experiences included feeling unable to respond adequately in social situations, feeling uncomfortable and pressured in one's interactions with others, feeling uncomfortable specifically related to making eye contact with others, generally feeling self-critical and inferior to others, feeling like others are especially focused on or watching/observing one, experiencing others as judging one negatively, intentionally hiding one's reactions in order to avoid exposure, and mistrusting or viewing others as threatening or intending one harm in some way. Related to this was a tendency to scrutinize oneself and feel self-conscious. 


\subsubsection{Unusual experiences of time (22 participants)}

Participants described numerous changes in their experiences of time, including both what might be characterized as implicit time (how time is experienced in the moment) as well as explicit time (how we reflect on our past or likely future; Fuchs \& van Duppen, 2017). This includes a variety of changes in immediate perception of passage of time and of the speed of one's own movements or the world, such as feeling like one moves more quickly or more slowly than others, or like time itself is flowing faster or slower than it seems like it should, or feeling like time is leaving one behind. Some described trouble organizing events that are happening in the present or in the past, a feeling that time has stopped or disappeared, as well as feeling like one is only to focus on or think about the present moment (and so unable to reflect on or integrate the past or the future). Some described feeling disconnected from the future: like the future might not occur, was irrelevant, or was impossible to affect with one's actions. Others found the future to be threatening and anxiety-provoking, or generally had a sense of being able to predict or anticipate future events. Some participants described a sense of past events feeling either stretched out and lasting longer than they actually did, or else having moved very quickly or being much more recent than reality.

\subsubsection{Changes in perception and sensation (23 participants)}

Participants described many different changes to sensation and perception. Many noted changes to the intensity of their sensations: sounds, tastes, textures, smells, colors, and general brightness could all feel more intense or less intense than usual. One's vision could be blurred or one could feel temporarily or partially blinded, sounds could seem distorted (especially human voices), or one may be especially sensitive to background noises. The qualities of sensory objects could also change: shapes may look different, the size of objects may appear larger or smaller than reality, apparent colors may change, and some textures may feel different (such as feeling dirty or less solid than reality). Some described these changes as seeming to emanate from the sensory objects themselves (e.g. music sounded louder), but many described the changes as related to their own sensory apparatus. Such changes tended to reflect a heightened sensitivity to various senses (especially vision, audition, and taste), though some noticed a tendency not to notice certain things (see also cognitive organization below). A number of participants also described changes to their ability to accurately or effectively perceive objects, including difficulty identifying the shape or sound that one is perceiving, difficulty identifying the direction or location of the origin of a sound (including feeling as though sounds were not coming from the object that produced them), and difficulty with the perceptual integrity: either feeling unable to perceive objects or landscapes as integrated wholes (rather than a set of isolated elements), or else difficulty seeing distinctions between separate objects. Specific changes to the perception of space were also noted, including problems with the accurate perception of distances (objects seeming further away or closer than they really were), feelings of disorientation (including getting lost in familiar places), a feeling of space expanding or else closing in, a feeling of objects or space being flattened, an increased awareness of space itself, and a sense of spaces and places having moods emanating from them.

\subsubsection{Confusion of the boundaries between self and world (22 participants)}


Participants described various feelings related to a blurring of the borders between self and nonself (either other people or, occasionally, objects). Some experienced a kind of privileged access to others' thoughts and private feelings, while other participants described the feeling that other people had control over their private thoughts or feelings, else that their thoughts or feelings were somehow public and exposed to others, or that close contact with others could infect or contaminate them in some way. A small number of participants described feeling like objects had merged with their body somehow, like objects or the world were communicating with them in some way, or that external sounds were coming from inside their head (or mind). Similarly, participants also described vivid experiences (similar or identical to perception) of things that are not actually/currently present. Some described this as having especially vivid imagination or visualization, others as having a very intense experience of past memories, some generally feeling confused about whether they were imagining or thinking something vs. perceiving or remembering it, and some noted that they could perceive sounds or images that were not actually present-either continuing to hear or see something that was previously, but no longer, present, or perceiving any number of percepts that were not present (including vague shapes, specific objects, geometric patterns, spots or dots, orbs of light, noises, voices, or smells).

\subsubsection{Disturbances of attention and cognitive organization (21 participants)}

Participants described several difficulties related to the ability to effectively attend to, think about, or remember things in order to achieve desired goals. This could include attentional difficulties, such as not noticing things that were obvious to others or else becoming distracted by irrelevant details or one's own thoughts, as well as being highly aware of repetitions or patterns of events or objects (for no apparent purpose), feeling as though one has too many thoughts or that thoughts are moving too quickly to be able to follow them effectively, feeling unable to effectively make decisions because of taking too many factors into consideration, and having difficulty remembering past events or remembering to do something in the future, which was often related to feeling present or attentive to the original experience or thought.

\subsubsection{Greater existential concerns about others and the world (17 participants)}

Some participants described a sense of stronger concern toward others and the world, or an experience of positive emotions associated with experiences. This could include a general sense of heightened connectedness to the humanity or the world, as well as feeling especially close to or familiar with people who the participant was in fact unfamiliar with, a stronger sense of empathy toward and understanding of others, a heightened sense of concern or responsibility for caring for other people or for the world/humanity as a whole, and a sense of being aware of and appreciating the beauty or other qualities of one's environment. These findings are in line with previous work highlighting greater existential concerns in people with depersonalization experiences, including concerns about the meaning of one's presence in the world, enhanced sense of observations of others' behavior and character, greater concern for the purpose and path of the humanity as a whole.

\subsection{Quantitative analysis}


A quantitative analysis was also conducted on the total IPASE scores. We conducted a four-stage hierarchical multiple regression with the total IPASE score as the dependent variable and the four CDS subscores (ABE, ASR, EN and AfS) as predictors. The subscales were entered in an order that seemed plausible given their relationship to self-perception, following (Farmer et al. 2020). Full results of the hierarchical regression are given in supplementary materials (see Table 2 ). While providing a detailed discussion of the quantitative analysis is beyond the scope of this paper, it is worth noticing that only the AfS was found to be a significant negative predictor of IPASE total score $(\beta=-0.755, p=0.007)$.

\begin{tabular}{lcccccc}
\hline \multicolumn{1}{c}{ Variable } & $\beta$ & $p$ & $\mathrm{R}^{2}$ & $\Delta \mathrm{R}^{2}$ & $\Delta \mathrm{F}$ & $\Delta \mathrm{p}$ \\
\hline Step 1 & & & 0.111 & 0.111 & 2.760 & 0.111 \\
Anomalous Body Experience & 0.334 & 0.111 & & & & \\
& & & & & & \\
Step 2 & & & 0.124 & 0.13 & 0.311 & \\
Anomalous Body Experience & 0.276 & 0.243 & & & & \\
Anomalous Subjective Recall & 0.128 & 0.583 & & & & \\
& & & & & & \\
Step 3 & & & 0.222 & 0.098 & 2.515 & \\
Anomalous Body Experience & 0.065 & 0.804 & & & & \\
Anomalous Subjective Recall & -0.062 & 0.809 & & & & \\
Emotional Numbing & 0.463 & 0.128 & & & & \\
& & & & & & \\
Step 4 & & & 0.476 & 0.254 & & \\
Anomalous Body Experience & 0.326 & 0.179 & & & & \\
Anomalous Subjective Recall & 0.041 & 0.849 & & & & \\
Emotional Numbing & 0.748 & 0.010 & & & & \\
Alienation from Surroundings & -0.755 & 0.007 & & & & \\
\hline
\end{tabular}

Table 2. Summary of the hierarchical regression analysis for CDS subscales predicting IPASE total scores.

\section{§ 4 Discussion}

Feelings of depersonalization (DP) are extremely common in the general population, yet underacknowledged and under-examined. Given that DP seems to affect the subjectivity of one's experiential life, inducing profound disruptions in self-awareness (Sierra and David 2011; Simeon and Abugel 2006), a phenomenological and qualitative approach may open a new window onto the very core of these experiences as lived from a first-person perspective.

In the current study we combined a well-established qualitative tool (IPASE) with an innovative tool (EAWE-SR) in order to provide key new insights into the experience of DP as lived from a 
first-person perspective. To the best of our knowledge, this is the first qualitative study examining anomalous self- and world-experiences in individuals with DP traits. As we will see shortly, exploring how DP affects not only (a)self-perception disturbances - that is, the way people relate to their own self and body - but also (b) world-perception disturbances - that is, the way people relate to the world and others - offers a richer and more complete picture of the phenomenon.

The descriptions from our participants with high levels of DP traits indicate the presence of a wide range of anomalous experiences, including those that are traditionally understood to be core features of depersonalization, as well as experiences that are not typically included in the depersonalization construct. Indeed, our findings may be clustered in two main groups: (a) anomalous self- and world-experiences usually associated to depersonalization traits and widely reported in previous research; (b) new experiential features that are less highlighted in previous work, and which may offer essential insights into the experience of DP, thus drawing attention to key aspects that otherwise may be overlooked for potential diagnosis.

In what follows we focus on the qualitative analysis and describe the main experiential clusters and the reported disturbances in turn, illustrating how our findings provide key new insights into the experience of depersonalisation as lived from a first-person perspective.

\subsection{Anomalous Bodily Experiences and Emotional Flatness}

In a comprehensive review of historical cases of pathological depersonalization, Sierra and Berrios (2001) found five major domains of experience that were consistent over time: changes in body experience, loss of agency, emotional numbing, changes in mental imagery and memory, and changes in visual perception of surroundings. Other studies (Sierra et al., 2005, Simeon et al., 2008) have found that these experiences separate into several distinct factors, including anomalous body experience, emotional numbing, anomalous subjective recall, and alienation from surroundings, with one study finding a fifth factor termed "body distortion" (Simeon et al., 2008). The results of the current study map onto these features and factors, particularly the changes in embodiment (which also includes disturbances of agency), detachment, affective changes, changes in perception and sensation, and cognitive organization (specifically related to disturbances of memory).

Anomalous bodily experiences have been consistently reported in relation to clinical and nonclinical depersonalization both in theoretical and experimental previous work (see Salami et al. 2020 for a recent discussion). Neuropsychological tests have noticed wide perceptual and attentional alterations in the pathophysiology of depersonalization-derealization (Guralnik et al., 2000, 2007). Impaired bodily self-awareness has been linked with disrupted physiological response in patients with DPD compared to healthy participants (Dewe et al. 2018; Owens et al. 2015; Sierra et al., 2002). DP has also been linked to disrupted activity in neural regions underlying somatic processing (Lemche, Brammer, et al., 2013; Lemche, Surguladze, et al., 2013; Medford et al., 2016). Recent studies found atypical integration of self-related visuotactile information (Adler et al. 2016; O’Sullivan et al. 2018; Farmer et al. 2020). Atypical neural 
activity have been reported in DPD patients with regards to sensory information (Medford et al., 2016) as well as regions responsible for the processing of visceral signals (Lemche et al., 2013). Interoception is typically defined as the perception and processing of visceral bodily signals, and the main brain regions responsible for processing interoceptive inputs are insula, cingulate cortex, amygdala and somatosensory cortex (Craig 2009). Crucially, all these regions have been linked with atypical activity on DP and DPD patients (Sierra et al. 2014; Medford 2012; Medford et al. 2016; Sierra et al. 2018). This led some theorists to examine links between disembodiment and interoceptive processing in DP (Michal et al. 2014; Seth et al. 2012; Gerrans 2018; Ciaunica et al. 2021a, b).

In our study, participants consistently reported feelings of 'disembodiment', and consequent emotional flatness and 'emptiness'. For example: "It feels like an out of body experience" (P216) or: "I feel like I'm watching from inside a shell. Everything besides my limbs feel like they don't have much of a presence" ( P 217). Even more strikingly: "I feel nothing. I just observe" (P 243) or: "People call me poker face. I often have trouble to show that I am happy from my tone or expression" (P215).

Although data from this study cannot demonstrate causality, previous research suggests that these feelings of disembodiment, and of being an 'onlooker' or 'watcher' or 'observer' of one's experiences are also impacting one's sense of agency: "I have a feeling of not actually doing something even though I am about to do it. I know what I am supposed to feel, but it does not come from me organically" (P 221). Lack of immersiveness or presence in the world makes people feel unreal, and seem to act like automata: "I sometimes feel like I'm not really present and I feel mechanical" (P124). Feelings of inner 'emptiness' are frequently reported: "I tend to feel as though it is empty inside and get tired from doing nothing" (P 153) or: "There's a physical feeling of emptiness in my chest" (P218) and: "I feel empty inside" (P248).

Previous work has proposed a link between the attenuation of the emotional response in DP and (a) decreased activities in emotional cortical (insula) and limbic (hypothalamus, amygdala), while (b) increased activities of the prefrontal cortex (Lemche et al., 2007, 2008; Medford et al., 2016; Sierra and David, 2011; Sierra et al., 2002b). Our findings again support the idea of a strong connection between anomalous bodily experiences and emotional numbing in DP. These experiences seem to be underpinned at the neural level by a decrease activity in limbic areas (consistent with the disembodiment and emotional flatness hypotheses) and an increase in prefrontal activity (consistent with the 'hyperreflexivity' hypothesis). We turn to this discussion now.

\subsection{Disturbances of the minimal self and hyperreflexivity}

Several theorists draw on a long-standing phenomenological tradition to define the minimal self as defined as the pre-reflective and immediate experience of one's body, actions, perceptions, and thoughts in relation to the world (Parnas and Sass 2003; Zahavi 2005; Fuchs 2005; Legrand 2006; Ciaunica 2016; Ciaunica \& Fotopoulou 2017). For example, Sass and Parnas (2003) have hypothesized that a basic self-disturbance (BSD) (also termed self-disorder, ipseity disturbance, or disturbance in the "minimal self") may lie at the core of severe psychiatric conditions such as 
schizophrenia and schizophrenia-spectrum disorders. Sass and Parnas (2003) have identified at two core features of BSD, namely: (a) "diminished self-affection or self-presence" which refers to a diminished sense of being immediately present to one's experiences; and (b) "hyperreflexivity" which refers to forms of heightened self-consciousness and heightened awareness of typically tacit or implicit aspects of one's experiences.

Much emphasis has been placed on diminished self-presence and altered self-awareness in DP (Sierra and David 2011). However, it also seems that hyperreflexivity may play a role in developing and maintaining DP symptoms. This over-scrutinization triggers simultaneously inner turmoil and non-responsiveness to the external world. Hence, diminished sense of self and presence and hyperreflexivity go hand in hand, like the two faces of the same coin (Fuchs 2005; Ciaunica et al. 2021b). Our study provides further support for this hypothesis. Indeed, our participants frequently reported these types of hyperreflexivity and existential turmoil. For example: "I sometimes feel separate from my own existence" (P124) or: "I'm not sure what the nature of existence might be, because all of the tools we would use to question it are part of this existence, so would naturally prove that we do, but how would we know if we were just the dream of a sleeping god or something?" (P241) and equally: "I feel that the universe has a special meaning and if I discover this meaning I will stop to exist. Sometimes I think people do not exist and even doubt about my existence at all, like if I were in a dream." (P236) or: Sometimes I'm so preoccupied with the way I walk or the way I present myself, I don't realize what's happening around me, for example if someone appeared next to me" (P218). These observations are in line with a wide body of research on DPD noting that individuals tend to focus on inner experiences and thoughts rather than attending and engaging with the external world (Hunter et al. 2003). Obsessional self-check of one's internal states: 'how do I feel now?', "Who am I"? DPD patients are drawn to ruminative and over-intellectualization of their inner workings (Hunter et al. 2003; Medford et al. 2005).

This hypothesis is further supported by the results of our quantitative analysis, suggesting that individuals with higher DP (as measured by the IPASE) show less alienation from surroundings (AfS, measured by the CDS). Speculatively, one may argue that this negative correlation underwrites a tendency in people experiencing a greater degree of self disturbance to be less aware of their surroundings, thus less likely to notice (or potentially experience) acute feelings of alienation from the world.

Previous phenomenological work has suggested that atypical self- and world-awareness in DP alters the transparency of basic, pre-reflective forms of self-awareness, which triggers consequent feelings of self-opacity and unrealness or lack of presence (Fuchs 2005; Ciaunica et al. 2020, 2021a,b). Transparency is an interesting and peculiar property of our experiences that can be intuitively grasped via the so-called 'window' metaphor. A perfectly clear and transparent window glass can give us the illusion of an unmediated access to a landscape, say. We typically take this transparency for granted, unless there is a 'crack' in the window, making the presence of the mediating glass noticeable (Ciaunica et al. 2020). The key idea embedded in this metaphor is that in DP, altered self-experiences or diminished self-presence - that is, the 'crack' in the transparent and typically unnoticed window of one's subjectivity - leads to hyperreflexivity. This form of enhanced awareness of typically tacit or implicit aspects of one's experiences trigger alienating feelings of 'opacity' are often and strikingly reported by DPD patients as 'having a pane of glass' or 'veil' interposed between one's self, body and the world, or "living in 
a bubble", or "feeling surrounded by an invisible wall" (Simeon \& Abugel, 2006; Ciaunica \& Charlton, 2018).

This over-scrutinization of one's inner workings may be accompanied by feelings of being 'trapped' in ones' head (mind) and outside one's body (Fuchs 2005; Ciaunica et al. 2020). Interestingly, our participants in this study frequently described similar feelings. For example: "I am very in my own head and sometimes so focused on myself, that I miss the surroundings or miss what is going on around me" (P136) or: "Sometimes I do feel like my mind has a mind of its own and does what it wishes while my body is not connected to it"(P136).

Taken together, these findings support the idea that a profound disruption of self-awareness lies at the heart of the depersonalization experiences as reported by previous research (Sierra \& David 2011; Seth et al. 2012; Billon 2016, 2017; Gerrans 2018). We also argue that depersonalization interferes with the transparency of one's minimal self, and that this is linked to feelings of unrealness, lack of presence, or distance between oneself and one's experiences. This lack of transparency was most obvious in the feelings of detachment described by most (though not all) participants, as well as in the feelings of disconnection from one's body and physical movements.

\subsection{Insights into overlooked features of depersonalization experiences}

Up to now we have examined our findings through the lens of previous work and hypotheses. We illustrated that the self-reports analyzed here support indeed the idea that anomalous bodily experiences, emotional flatness and consequent alterations of the minimal self and hyperreflexivity be considered as core features of DP experiences.

Interestingly, though, it is worth noting that there were many features reported by participants that are not described in the literature on depersonalization experiences, and that are not captured in common measures of depersonalization like the CDS. For example, various forms of 'blurriness' or confusion between oneself and the world, such as being unsure about whether something was objectively perceived or simply imagined or thought, were described by numerous participants. This confusion extends also to the presence or even the realness of other people: "A few times I've had the experience of being in a crowd of people, and feeling as though some of them were zombies or clones or mannequins, or something like that. It's an eerie feeling and I know that they are people, but I have to watch them for a little while to make sure they do something human- like. Sometimes it feels as though people's movements are programmed or studied versions of human movements" (P241).

Similarly, many participants also described disturbances in temporal organization, particularly with ordering events that occurred in the past. For example, "The last week in my memory is a few events with no connection, sometimes I really can't imagine what else I did in the last day/week" (P221). Temporal perception seems here to be of particular interest, with many participants reporting that the time seems to fly faster than before experiencing DP, e.g. "Sometimes I feel like time is moving incredibly fast and I lose the perception of time" (P136). 
We also found alterations in goal-directed thinking and attention (cognitive organization), described by one participant as, "Very often, I only hear half of what is said to me. I try very hard to bring myself in the here and now. My mind wanders away without my control really" (P220). Another stated, "Especially with my thoughts, they seem to jump about and miss steps out and I can get very lost within them" (P241).

Importantly, we noticed a heighted sensitivity to various sensations from different sensory modalities. One participant stated, "I find myself wincing from light colored things especially" (P221), while another noted, "The voice of those around me sometimes seems so loud that my ears pulsate" (P201).

It would be interesting and important to examine more closely in future work both the sensitivity to distinct sensory inputs (visual, auditory, tactile, olfactory, vestibular, proprioceptive) and their impact on time and space perception in people with DP experiences. Specifically, it would be key to investigate to what extent feelings of (un)realness are triggered by atypical sensorimotor integration of self- and world-related information. For example, it has been recently suggested that different senses and modes of perception (e.g. vision versus touch) may provide a different basis for self- and world-experiences (Ciaunica et al. 2021a). Distant visuospatial perception such as seeing/recognizing oneself in the mirror - may enhance feelings of estrangement and self-detachment, of being inside one's head. While active engagements with the world and others via proximal (tactile and multisensory) interactions may enhance the sense of self, realness and presence in people with DPD. This is because, paradoxically, in order to get closer to one's self, one needs to be able to 'forget' oneself (Ciaunica et al. 2021b) and to be able to open to the world and others, via proximal tactile interactions.

As one person with lived experiences of DP strikingly puts the point: "When the depersonalisation is very deep, I still seek to 'be' someone else because it feels like that constant source of interaction is the only thing that allows me to maintain a connection with the world. I'll also seek physical contact with whoever I'm with. It almost feels as though I need to be that other person because my own sense of self is not strong enough in that moment to sustain me." (Ciaunica \& Charlton 2018). Indeed, this observation may be key for potential interventions and therapies designed to make DP people more aware of what still connects them with their self, body and the world, as opposed to focusing on what disconnects them from one's self and others. Specifically, future work needs to disentangle and contrast the role of proximal (touch, interoception) versus distal (vision) senses in shaping the experience of being a self present in the world and fully connected with one's body.

Participants also described many disturbances related to self-concept and to relationships with others, including feelings of inferiority and insecurity, feeling uncomfortable around or exhausted by others, feeling disconnected from others, and adapting one's behavior to elicit others' approval; as well as more profound changes such as uncertainty about one's identity and lack of continuity in time. For example: "I can't connect the me right now to other 'me's throughout time" (P217). 
Indeed, our study suggests that no domain of experience may go untouched: the passage of time, relationships with others, language and communication, attention and cognitive organization, and boundaries between self and non-self all become more blurred, more disorganized, less transparent, and less functional. Furthermore, thoughts and beliefs about oneself and others also seem to be deeply affected, as participants frequently felt insecure, self-critical, and uncomfortable, anxious, or mistrustful in social situations. Crucially, mood and affect were also altered beyond typical descriptions of diminished affective response, as participants described profound feelings of hopelessness and demoralization as well as high levels of anxiety and restlessness.

Overall, our results suggest that there may be many more experiential features that are relevant to understanding and assessing depersonalization than are typically elicited or discussed in research and clinical and non-clinical settings. In addition, they indicate potentially important targets for interventions for people with DP experiences; and treatment of persons with DPDR. Our findings also point to a need for additional research to specify the nature of their relationship to the depersonalization range of experiences and DPD as a clinical syndrome.

\section{§ 5 Limitations and Future Directions}

Our study presents several important limitations. First, the participants included in this study were only identified as having high levels of trait depersonalization, and therefore their diagnostic status is unknown. This may make it difficult to generalize the experiences reported here to those of persons who meet criteria for DPD. However, the fact that most participants have subthreshold levels of depersonalization suggests that individuals who do meet criteria for DPD (and who therefore are likely to have more extreme symptoms and clinical features) are likely to also experience a vast range of experiential anomalies, perhaps of even greater number and severity.

Second, participants were not assessed for presence of a schizophrenia-spectrum diagnosis, therefore it is possible that some participants might have met criteria for schizophrenia or a related disorder. At the same time, it is highly unlikely that all (if any) participants who expressed such experiences have an underlying schizophrenia-spectrum condition or vulnerability, and this data suggests that such similarities should not be ruled out. Future research will need to develop a clearer understanding of the relationship between schizophrenia, depersonalization, and disturbances of basic selfhood.

Also, due to the self-report nature of these comments, crucial details that might affect the understanding of a participant's statement may not always have been present in the same way that might be elicited by an in-depth interview. Therefore, analysis erred on the side of caution when assigning codes and themes, and left out any statements that could be argued as representing a commonplace or typical form of experience (rather than assuming a more dramatic alteration). The use of self-reported scales is associated with other various limitations: (1) the answers are dependent on the honesty of participants; (2) the use of scales with subjective questions may difficult its understanding/interpretation and the introspective ability to provide an 
accurate answer; and (3) its use may have skewed our population, as people prone to answering questionnaires, and therefore with different personal characteristics, are more likely to have answered it. We believe that the benefit of collecting data from a relatively large sample on a large number of potential experiences is worth the loss that comes from somewhat limited selfreport comments. However, future research would benefit from conducting in-depth interviews on a similar range of topics with more participants.

Intriguingly, feelings of being a neutral observer of one's perceptions, emotions and actions has been reported in connexion with contemplative practices such as meditation (Lindahl \& Britton 2019; Ciaunica et al. 2020). For example, in our study, the participants similarly described feelings of self-split or being an 'external' observer : "Sometimes I feel like I'm a watching a movie of myself" (P218). Or "I also feel like the me who speaks is somewhat separate to the me who thinks" (P217). Future work needs to examine and to disentangle the positive versus negative valence of these feelings of self-detachment as reported in depersonalisation and in meditation, as well as their underlying neural mechanisms and phenomenological markers.

Finally, further work needs to combine qualitative phenomenological tools, with quantitative behavioural and neural measures in order to provide an integrative examination of depersonalisation experiences, providing thereby a much needed rich and multifaceted approach to this neglected yet widespread condition. 


\section{References}

- Adler, J., Schabinger, N., Michal, M., Beutel, M. E., \& Gillmeister, H. (2016). Is that me in the mirror? Depersonalisation modulates tactile mirroring mechanisms. Neuropsychologia, $85,148-158$.

- American Psychiatric Association. (2013). Diagnostic and statistical manual of mental disorders (Fifth ed.). Washington, D.C.: American Psychiatric Association.

- Armour, C., Contractor, A. A., Palmieri, P. A., \& Elhai, J. D. (2014). Assessing Latent Level Associations Between PTSD and Dissociative Factors: Is Depersonalization and Derealization Related to PTSD Factors More So than Alternative Dissociative Factors? Psychological Injury and Law, 7(2), 131-142.

- $\quad$ Baker, D., Hunter, E. C. M., Lawrence, E., Medford, N., Patel, M., Senior, C., ... David, A. S. (2003). Depersonalisation disorder: Clinical features of 204 cases. British Journal of Psychiatry, 182, 428-433.

- Billon, A. (2016), 'Making Sense of the Cotard Syndrome: Insights from the Study of Depersonalisation', Mind and Language, 31: 356-91.

- Billon, A. (2017). 'Mineness First: three challenges to recent theories of the sense of bodily ownership' in A. Alsmith and F. de Vignemont (eds) The Subject's Matter: SelfConsciousness and the Body. Cambridge MA: MIT Press.

- Bliss E. L. (1959). "Studies of Sleep Deprivation-Relationship to Schizophrenia.” Archives of Neurology \& Psychiatry 81: 348-359.

- Braun, V., \& Clarke, V. (2006). Using thematic analysis in psychology. Qualitative Research in Psychology, 3(2), 77-101.

- Blanke, O., and Metzinger, T. (2009). Full-body illusions and minimal phenomenal selfhood. Trends in Cognitive Science, 13, 7-13.

- Ciaunica, A. (2016). Basic Forms of Pre-reflective Self-Consciousness: a Developmental Perspective. In Pre-reflective Self-Consciousness: Sartre and Contemporary Philosophy of Mind, eds. S. Miguens, G. Preyer, and C. Morando, 422-438. London: Routledge.

- Ciaunica, A. \& Fotopoulou, A. (2017). 'The Touched Self: Psychological and Philosophical Perspectives on Proximal Intersubjectivity and the Self'. In Durt C., Fuchs T., and Tewes C. (eds). Embodiment, Enaction, and Culture-Investigating the Constitution of the Shared World. Cambridge MA: MIT Press, p. 173-192.

- Ciaunica, A., Charlton, J., 2018. When the self slips: what depersonalization can say about the self - https://aeon.co/essays/what-can-depersonalisation-disorder-say-about-the-self

- Ciaunica, A., Charlton, J. \& Farmer, H. (2020) - When the Window Cracks: Transparency and the Fractured Self in Depersonalisation. Phenomenology and Cognitive Science https://doi.org/10.1007/s11097-020-09677-z

- Ciaunica, A., Petreca, B., Fotopoulou, A., \& Roepstorff, A. (2021a, in press). Whatever Next and Close to my Self - The Transparent Senses and the 'Second Skin': Implications for the Case of Depersonalisation. Frontiers in Psychology.

- Ciaunica, A., Hesp, C., Seth, A., Limanowski, J., \& Friston, K. (2021b, under review). I overthink - therefore I am not: Altered Sense of Self in Depersonalisation Disorder. Preprint here : https://doi.org/10.31234/osf.io/k9d2n

- Cicero, D. C., Neis, A. M., Klaunig, M. J., \& Trask, C. L. (2017). The inventory of Psychotic-like Anomalous Self-Experiences (IPASE): Development and validation. Psychological Assessment, 29(1), 13-25. 
- Clark, L. A., \& Watson, D. (1995). Constructing validity: Basic issues in objective scale development. Psychological Assessment, 7(3), 309-319.

- Conerty, J., Skodlar, B., Pienkos, E., Zadravek, T., Byrom, G., \& Sass, L. (2017). Examination of anomalous world experience: A report on reliability. Psychopathology, 50(1), 55-59.

- Craig, A. D. (2009). How do you feel-now? The anterior insula and human awareness. Nature Reviews Neuroscience, 10, 59-70.

- Devinsky O, Putnam F, Grafman J, Bromfield E, Theodore WH. Dissociative states and epilepsy. Neurology. 1989 Jun;39(6):835-40.

- Dewe, H., Watson, D. G., Kessler, K., \& Braithwaite, J. J. (2018). The depersonalized brain: New evidence supporting a distinction between depersonalization and derealization from discrete patterns of autonomic suppression observed in a non-clinical sample. Consciousness and Cognition, 63: 29-46.

- Dixon, J., 1963. Depersonalization phenomena in a sample population of college students. Br. J. Psychiatry 109, 371-375.Dugas L. and Moutier F. (1911).La Dépersonnalisation. Paris, Felix Alean References

- $\quad$ Farmer, H., Cataldo, A., Adel, N., Wignall, E., Gallese, V., Deroy, O., Hamilton, A., \& Ciaunica, A. (2020). The Detached Self: Investigating the Effect of Depersonalisation on Self-Bias in the Visual Remapping of Touch, Multisensory Research, 1-22.

- Fleming G. W. T. H. (1936). "Mescaline and Depersonalization." (Journal of Neurology and Psychopathy xvi: 193).

- Friston, K. J. (2017). Precision Psychiatry. Biological Psychiatry-Cognitive Neuroscience and Neuroimaging, 2(8): 640-643.

- Fuchs, T., \& Van Duppen, Z. (2017). Time and events: On the phenomenology of temporal experience in schizophrenia (ancillary article to eawe domain 2). Psychopathology, 50(1), 68-74.

- Fuchs T (2005) Corporealized and disembodied minds. A phenomenological view of the body in melancholia and schizophrenia. Philos Psychiatry Psychol 12:95-107

- Fuchs, T. (2015). From self-disorders to ego disorders. Psychopathology, 48(5), 324-331.

- Gallagher, S. (2000). Philosophical conceptions of the self: implications for cognitive science. Trends in cognitive sciences, 4(1), 14-21.

- Gerrans, P., 2019. Depersonalization disorder, affective processing and predictive coding. Rev. Philos. Psychol. 10, 401-418.

- Guralnik, O., Schmeidler, J., Simeon, D., 2000. Feeling unreal: cognitive processes in depersonalization. Am. J. Psychiatry 157, 103-109.

- Guralnik, O., Giesbrecht, T., Knutelska, M., Sirroff, B., Simeon, D., 2007. Cognitive functioning in depersonalization disorder. J. Nerv. Ment. Dis. 195, 983-988.

- Harper, M., Roth, M., 1962. Temporal lobe epilepsy and the phobic anxietydepersonalization syndrome: I: a comparative study. Compr. Psychiatry 3, 129-151.

- Henriksen, M. G., Škodlar, B., Sass, L. A., \& Parnas, J. (2010). Autism and perplexity: A qualitative and theoretical study of basic subjective experiences in schizophrenia. Psychopathology, 43(6), 357-368.

- Hohwy, J. (2007) The Sense of Self in the Phenomenology of Agency and Perception, Psyche 13 (1), pp. 1-20.

- Hunter, E.C., Sierra, M., David, A.S., 2004. The epidemiology of depersonalization and derealisation. A systematic review. Society of Psychiatry Psychiatric Epidemiology39: 9-18. 
- Hunter EC., Sierra M, David AS (2004) .The epidemiology of depersonalization and derealisation. A systematic review. Society of Psychiatry Psychiatric Epidemiology, 39: 918.

- Hunter, E.C., Charlton, J., David, A.S., 2017. Depersonalisation and derealisation: assessment and management. BMJ 356, j745.

- Kaplan, H.I., Sadock, B.J., Grebb, J., 1998. Substance related disorders. Kaplan H.I., Sadock B.J. Kaplan and Sadock's Synopsis of Psychiatry: Behavioral Sciences, Clinical Psychiatry, 8th ed. Williams \& Wilkins, Baltimore, pp. 419-426.

- Kraus A. (2002). "Melancholie: Eine art von depersonalisation. Affekt und affektive Stoerungen." In T. Fuchs and C. Mundt (eds.) Affekt und affektive Storungen, pp. 169-186. Paderborn: Schoningh.

- Langfeldt G. (1960). "Diagnosis and Prognosis of Schizophrenia." Proc R Soc Med 53: $1047-1052$.

- Lambert MV, Sierra M, Phillips ML, David AS. The spectrum of organic depersonalization: a review plus four new cases. J Neuropsychiatry Clin Neurosci. 2002 Spring;14(2):141-54.

- Lanius RA, Brand B, Vermetten E, Frewen PA, Spiegel D. The dissociative subtype of posttraumatic stress disorder: rationale, clinical and neurobiological evidence, and implications. Depress Anxiety. 2012 Aug;29(8):701-8. doi: 10.1002/da.21889.

- Legrand, D. (2006). The bodily self: The sensorimotor roots of pre-reflective selfconsciousness. Phenomenology and the Cognitive Sciences, 5(1): 89-118.

- Lemche, E., Brammer, M. J., David, A. S., Surguladze, S. A., Phillips, M. L., Sierra, M., ... Giampietro, V. P. (2013). Interoceptive-reflective regions differentiate alexithymia traits in depersonalization disorder. Psychiatry Research - Neuroimaging, 214(1), 66-72.

- Lemche E, Surguladze SA, Brammer MJ, Phillips ML, Sierra M, David AS, Williams SC, Giampietro VP (2016). Dissociable brain correlates for depression, anxiety, dissociation, and somatization in depersonalization-derealization disorder. CNS Spectr. 2016 Feb;21(1):35-42.

- Limanowski, J. and Friston, K., (2018). 'Seeing the dark': Grounding phenomenal transparency and opacity in precision estimation for active inference. Frontiers in psychology, 9, p.643.

- Madeira, L., Pienkos, E., Filipe, T., Melo, M., Queiroz, G., Eira, J., . . Sass, L. (2019). Self and world experience In non-affective first episode of psychosis. Schizophrenia Research, 211, 69-78. doi:10.1016/j.schres.2019.07.001

- Medford, N. (2012). Emotion and the unreal self: Depersonalization disorder and deaffectualization. Emotion Review, 4(2), 139-144. doi:10.1177/1754073911430135

- Medford, N., Sierra, M., Baker, D., \& David, A. S. (2005). Understanding and treating depersonalisation disorder. Advances in Psychiatric Treatment, 11(2), 92-100. doi:10.1192/apt.11.2.92

- Mayer-Gross W. (1935). “On Depersonalization1.” British Journal of Medical Psychology 15: 103-126.

- Merleau-Ponty, M. (1945/1962). Phénoménologie de la perception, Paris: Éditions Gallimard; English translation: C. Smith. Phenomenology of Perception, London: Routledge and Kegan Paul, 1962.

- Metzinger, T. (2003). Being No One: The Self-Model Theory of Subjectivity. Cambridge, MA: MIT Press.

- Michal M, Beutel ME, Grobe TG (2010). Wie oft wird die DepersonalisationsDerealisationsstörung (ICD-10: F48.1) in der ambulanten Versorgung diagnostiziert? [How 
often is the Depersonalization-Derealization Disorder (ICD-10: F48.1) diagnosed in the outpatient health-care service?]. Z Psychosom Med Psychother. 2010;56(1):74-83.

- Nelson, B., Li, E., Cicero, D. C., Gawęda, Ł, Hartmann, J. A., Koren, D., . . Lavoie, S. (2018). The construct validity of the inventory of PSYCHOTIC-LIKE ANOMALOUS Selfexperiences (IPASE) as a measure of MINIMAL self-disturbance: Preliminary data. Early Intervention in Psychiatry, 13(3), 686-691.

- Nelson, B. (2013), Varieties of self-disturbance. Early Intervention in Psychiatry, 7: 231234. https://doi.org/10.1111/eip.12080

- Nordgaard, J., \& Parnas, J. (2014). Self-disorders and the schizophrenia spectrum: A study of 100 first hospital admissions. Schizophrenia Bulletin, 40(6), 1300-1307. doi:10.1093/schbul/sbt239

- $\quad$ Owens, A. P., David, A. S., Low, D. A., Mathias, C. J., \& Sierra-Siegert, M. (2015). Abnormal cardiovascular sympathetic and parasympathetic responses to physical and emotional stimuli in depersonalization disorder. Frontiers in Neuroscience, 9, 89.

- O’Sullivan, N., de Bezenac, C., Piovesan, A., Cutler, H., Corcoran, R., Fenyvesi, D., \& Bertamini, M. (2018). I am there ... but not quite: An unfaithful mirror that reduces feelings of ownership and agency. Perception, 47(2), 197-215.

- Parnas, J., \& Handest, P. (2003). Phenomenology of anomalous self-experience in early schizophrenia. Comprehensive Psychiatry, 44(2), 121-134. doi:10.1053/comp.2003.50017

- Parnas, J., Møller, P., Kircher, T., Thalbitzer, J., Jansson, L., Handest, P., \& Zahavi, D. (2005). EASE: Examination of anomalous self-experience. Psychopathology, 38, 236-258.

- Parnas, J., Raballo, A., Handest, P., Jansson, L., Vollmer-Larsen, A., \& Sæbye, D. (2011). Self-experience in the early stages of schizophrenia: 5-year follow-up of the Copenhagen prodromal study. World Psychiatry, 10(3), 200-204.

- Pienkos, E. (2020). Schizophrenia in the world: Arguments for a contextual phenomenology of psychopathology. Journal of Phenomenological Psychology, 51, 184-206.

- Pienkos, E., Silverstein, S.,\& Sass, L. (2017). The phenomenology of anomalous world experience in schizophrenia: A qualitative study. Journal of Phenomenological Psychology, 48, 188-213.

- Qin P, Wang M, Northoff G. Linking bodily, environmental and mental states in the self-A three-level model based on a meta-analysis. Neurosci Biobehav Rev. 2020 Aug;115:77-95.

- Raballo, A. \& Parnas, J. (2011). The silent side of the spectrum: Schizotypy and the schizotaxic self. Schizophrenia Bulletin, 37(5), 1017-1026.

- Salami A, Andreu-Perez J, Gillmeister H. Symptoms of depersonalisation/derealisation disorder as measured by brain electrical activity: A systematic review. Neurosci Biobehav Rev. 2020 Nov;118:524-537

- $\quad$ Sass. L., Pienkos, E., Nelson, B., \& Medford, N. (2013). Anomalous self-experience in depersonalization and schizophrenia: A comparative investigation. Consciousness and Cognition, 22, 430-441.

- Sass, L. \& Pienkos, E. (2015). Faces of intersubjectivity: A phenomenological study of interpersonal experience in melancholia, mania, and schizophrenia. Journal of Phenomenological Psychology, 46, 1-32. 
- Sass, L. A. \& Parnas, J. (2003). Schizophrenia, consciousness, and the self. Schizophrenia Bulletin, 29(3), 427-444. doi: 10.1093/oxfordjournals.schbul.a007017

- Sass, L., Pienkos, E., Skodlar, B., Stanghellini, G., Fuchs, T., Parnas, J., \& Jones, N. (2017). EAWE: Examination of anomalous world experience. Psychopathology, 50, 10-54. doi: $10.1159 / 000454928$

- $\quad$ Sass, L., Borda, J. P., Madeira, L., Pienkos, E., \& Nelson, B. (2018). Varietes of self disorder: A bio-pheno-social model of schizophrenia. Schizophrenia Bulletin, 1-8.

- Serra L., Fadda L., Buccione I., Caltagirone C., and Carlesimo G. A. (2007). "Psychogenic and Organic Amnesia. A Multidimensional Assessment of Clinical, Neuroradiological, Neuropsychological and Psychopathological Features." Behavioural Neurology 18: 53-64.

- Seth, A.K., Suzuki, K. \& Critchley, H.D. (2011).An interoceptive predictive coding model of conscious presence. Frontiers in Psychology, 2, 395.

- Sierra, M. (2009). Depersonalization: A new look at a neglected syndrome. Cambridge: Cambridge University Press.

- $\quad$ Sierra, M., Baker, D., Medford, N., \& David, A. S. (2005). Unpacking the depersonalization syndrome: An exploratory factor analysis on the Cambridge Depersonalization Scale. Psychological Medicine, 35, 1523-1532.

- Sierra, M. \& Berrios, G. E. (1997). Depersonalization: A conceptual history. History of Pyshciatry, 8, 213-229.

- Sierra, M., \& Berrios, G. E. (2000). The Cambridge Depersonalisation scale: A new instrument for the measurement of depersonalisation. Psychiatry Research, 93(2), 153-164.

- Sierra, M. \& Berrios, G. E. (2001). The phenomenological stability of depersonalization: Comparing the old with the new. The Journal of Nervous and Mental Disease, 189(9), 629636.

- Sierra, M. \& David, A. S. (2011). Depersonalization: A selective impairment of selfawareness. Consciousness and Cognition, 20, 99-108.

- Simeon, D., \& Abugel, J. (2006). Feeling Unreal: Depersonalization Disorder and the Loss of the Self. Oxford: Oxford University Press.

- $\quad$ Simeon, D., Guralnik, O., Hazlett, E. A., Spiegel-Cohen, J., Hollander, E., \& Buchsbaum, M. S. (2000). Feeling unreal: A PET study of depersonalization disorder. American Journal of Psychiatry, 157(11): 1782-1788.

- $\quad$ Simeon, D., Knutelska, M., Nelson, D., \& Guralnik, O. (2003). Feeling Unreal: A Depersonalization Disorder Update of 117 Cases. The Journal of Clinical Psychiatry, 64(9): 990-997.

- $\quad$ Simeon, D., Kozin, D. S., Segal, K., Lurch, B., Dujour, R., \& Giesbrecht, T. (2008). Deconstructing depersonalization: Further evidence for symptom clusters. Psychiatry Research, 157, 303-306.

- Stanghellini, G. \& Ballerini, M. (2007). Values in persons with schizophrenia. Schizophrenia Bulletin, 33(1), 131-141.

- Stanghellini, G. \& Ballerini, M. (2011). What is it like to be a person with schizophrenia in the social world? A first-person perspective study on schizophrenia dissociality- Part 2: Methodological issues and empirical findings. Psychopahtology, 44, 183-192. Tibubos, A.N., Grammes, J., Beutel, M.E., Michal, M., Schmutzer, G., Brähler, E., 2018.Emotion regulation strategies moderate the relationship of fatigue with depersonalization and derealization symptoms. J. Affect. Disord. 227, 571-579. 
- van Heugten-van der Kloet, D., Giesbrecht, T., Merckelbach, H., 2015. Sleep loss increases dissociation and affects memory for emotional stimuli. J. Behav. Ther. Exp. Psychiatry 7, 917.

- Værnes, T. G., Røssberg, J. I., \& Møller, P. (2018). Anomalous self-experience: Markers of schizophrenia vulnerability or symptoms of depersonalization disorder? A phenomenological investigation of two cases. Psychopathology, 51, 198-209.

- Zahavi, D., (2005). Subjectivity and Selfhood: Investigating the first-person perspective, Cambridge, MA: The MIT Press. 